\title{
Risk Assessment of Posthepatectomy Liver Failure Using Hepatobiliary Scintigraphy and CT Volumetry
}

\author{
Sander Dinant ${ }^{1}$, Wilmar de Graaf ${ }^{1}$, Bart J. Verwer ${ }^{1}$, Roelof J. Bennink ${ }^{2}$, Krijn P. van Lienden ${ }^{3}$, Dirk J. Gouma ${ }^{1}$, \\ Arlène K. van Vliet ${ }^{1}$, and Thomas M. van Gulik ${ }^{1}$ \\ ${ }^{1}$ Department of Surgery, Academic Medical Center, Amsterdam, The Netherlands; ${ }^{2}$ Department of Nuclear Medicine, Academic Medical \\ Center, Amsterdam, The Netherlands; and ${ }^{3}$ Department of Radiology, Academic Medical Center, Amsterdam, The Netherlands
}

\begin{abstract}
A major part of morbidity and mortality after liver resections is caused by inadequate remnant liver function leading to liver failure. It is therefore important to develop accurate diagnostic tools that can predict the risk of liver resection-related morbidity and mortality. In this study, preoperative hepatobiliary scintigraphy of the future remnant liver and CT volumetric measurement of the future remnant liver were performed on patients who were to undergo liver resection. The accuracy of risk assessment for postoperative morbidity, liver failure, and mortality was evaluated. Methods: Forty-six patients who were scheduled for liver resection because of hepatobiliary tumors, including 17 patients with parenchymal disease (37\%) and 13 patients with hilar cholangiocarcinoma (28\%), were assessed preoperatively. Hepatobiliary scintigraphy was performed by drawing regions of interest around the future remnant to calculate ${ }^{99 \mathrm{~m}} \mathrm{Tc}$-mebrofenin uptake in it. CT volumetry was used to measure the volume of the total liver, the tumors, and the future remnant. Receiver-operatingcharacteristic analysis was performed to assess cutoff values for risk assessment of morbidity, liver failure, and mortality. Furthermore, univariate and multivariate analyses were performed to determine factors related to morbidity and mortality. Results: Morbidity and mortality rates were $61 \%$ and $11 \%$, respectively. Liver failure occurred in 6 patients (13\%). Significantly decreased uptake in the future remnant was found in patients in whom liver failure and liver failure-related mortality developed $(P=0.003$ and 0.02 , respectively). The volume of the future remnant was not significantly associated with any of the outcome parameters. In receiver-operating-characteristic analysis, uptake cutoff values for liver failure and liver failure-related mortality were $2.5 \% / \mathrm{min} /$ body surface area and $2.2 \% / \mathrm{min} /$ body surface area, respectively. In multivariate analysis, uptake was the only significant factor associated with liver failure. Conclusion: Preoperative measurement of ${ }^{99 \mathrm{mTc}-m e b r o f e n i n}$ uptake in the future remnant liver on hepatobiliary scintigraphy proved more valuable than measurement of the volume of the future remnant on CT in assessing the risk of liver failure and liver failure-related mortality after partial liver resection.
\end{abstract}

\footnotetext{
Received Nov. 28, 2006; revision accepted Feb. 12, 2007.

For correspondence or reprints contact: Sander Dinant, PhD, Surgical Laboratory, Department of Surgery, IWO-1-173, Academic Medical Center, Meibergdheef 9, 1105A2, Amsterdam, The Netherlands.

E-mail: s_dinant@hotmail.com

COPYRIGHT @ 2007 by the Society of Nuclear Medicine, Inc.
}

Key Words: iminodiacetic acid; hepatobiliary scintigraphy; CT volumetry; liver failure; hepatectomy

J Nucl Med 2007; 48:685-692

DOI: 10.2967/jnumed.106.038430

$\mathbf{D}_{\text {e }}$ espite newer techniques such as radiofrequency ablation and arterial chemoembolization, partial liver resection remains the treatment of choice for most primary and secondary liver malignancies. Although overall mortality rates have improved in the last decade, postoperative morbidity rates still can be substantial. A large resection and underlying parenchymal disease such as cirrhosis or obstructive jaundice correlate with increased morbidity and mortality rates $(1-6)$. When too much healthy liver parenchyma is resected, the risk of liver failure increases. Liver failure is an important cause of mortality after partial liver resection $(2,6)$. For patients with healthy livers, a resection of $70 \%-$ $75 \%$ of the liver is assumed to be safe (7). For patients with parenchymal disease, this safety limit is more variable and lies between $40 \%$ and $60 \%$. CT volumetry can be used to calculate the volume of the future remnant liver (8) and is widely used to exclude patients from liver resection or to select patients who will benefit from a procedure to increase the volume of the future remnant, such as portal vein embolization (9). However, the function of the future remnant is decreased in patients with parenchymal disease, despite a future-remnant volume that is equal to that in patients with healthy livers $(7,10,11)$. Therefore, one can argue that determining the functional capacity of the future remnant is more important than determining the volume, especially in patients with parenchymal disease.

Hepatobiliary scintigraphy using ${ }^{99 \mathrm{~m}} \mathrm{Tc}$-iminodiacetic acid analogs, such as ${ }^{99 m}$ Tc-mebrofenin, can be used to measure segmental liver function $(12,13)$. ${ }^{99 m}$ Tc-Mebrofenin is excreted into the bile by adenosine triphosphate-dependent export pumps - the multidrug-resistance-associated proteins 1 and 2 -without undergoing biotransformation during transit through the hepatocytes (14). We have shown that the 
preoperative total hepatic uptake rate of ${ }^{99 \mathrm{~m}} \mathrm{Tc}$-mebrofenin correlates well with the indocyanine green (ICG) clearance rate (15). Furthermore, ${ }^{99 \mathrm{~m} T c-m e b r o f e n i n ~ u p t a k e ~ b y ~ t h e ~}$ future remnant can be determined preoperatively by delineating the future remnant on the preoperative hepatobiliary scintigraphy image. In a previous study, a strong correlation was found between the uptake rate of the future remnant and the actually measured uptake $1 \mathrm{~d}$ after surgery $(r=0.95$; $P<0.001)(16)$.

The aim of this study was to measure uptake in the future remnant liver preoperatively on ${ }^{99 \mathrm{~m}} \mathrm{Tc}-$ mebrofenin hepatobiliary scintigraphy, measure the volume of the future remnant liver on CT volumetry, and compare the accuracies of these 2 methods in predicting the outcome of liver resections in a series including patients with parenchymal disease and hilar cholangiocarcinoma. The outcome parameters were postoperative morbidity, liver failure, and mortality.

\section{MATERIALS AND METHODS}

\section{Patients}

Forty-six patients (23 men and 23 women; median age, 63 y; range, 19-79 y) who underwent a partial liver resection from 2000 to 2004 were consecutively included in this study. All patient data used in the study of Bennink et al. (16) were included in our study. Postoperative histopathologic examination showed the following diagnoses: colorectal metastasis $(n=16)$, hilar cholangiocarcinoma $(n=13)$, hepatocellular carcinoma $(n=6)$, chronic sclerosing cholangitis $(n=4)$, cystadenoma $(n=2)$, metastasis of adenosarcoma of the uterus $(n=1)$, metastasis of nonseminoma testis $(n=1)$, cholangiocellular carcinoma $(n=1)$, metastasis of gastrinoma $(n=1)$, and focal nodular hyperplasia $(n=1)$. A total of 17 patients $(37 \%)$ had histologically proven parenchymal disease, of which 11 had fibrosis, 2 cirrhosis, and 4 chronic sclerosing cholangitis. The study was approved by the Medical Ethics Committee of the Academic Medical Center of the University of Amsterdam. Written informed consent was obtained from each patient before participating in the study.

\section{Preoperative Workup}

The routine preoperative workup consisted of physical examination and laboratory analysis of liver damage parameters and function parameters. These parameters were also measured at regular intervals after surgery. Furthermore, the ICG clearance rate was measured as described elsewhere (15). In cholestatic patients $(n=$ 18), preoperative biliary drainage was performed using endoscopic retrograde cholangiopancreatography $(n=15)$ or percutaneous transhepatic drainage $(n=3)$ more than $6 \mathrm{wk}$ before surgery. Overall, preoperative total bilirubin levels were $15 \pm 12 \mu \mathrm{mol} / \mathrm{L}$ (mean \pm $\mathrm{SD})$. One day before surgery, liver damage and function parameters were analyzed, ICG clearance rate measured, and hepatobiliary scintigraphy performed.

\section{Hepatobiliary Scintigraphy}

Hepatobiliary scintigraphy was performed using the radiopharmaceutical agent ${ }^{99 \mathrm{~m}} \mathrm{Tc}$-mebrofenin as previously described (16). Briefly, after injection of $85 \mathrm{MBq}$ of $99 \mathrm{~m} \mathrm{Tc}-$ mebrofenin, dynamic images were obtained with a $\gamma$-camera (Diacam; Siemens) for 60 min. During the first $10 \mathrm{~min}, 60$ frames of $10 \mathrm{~s}$ each were acquired (liver uptake phase), followed by 50 frames of 1 min each (liver excretion phase). On preoperative scintigraphy, regions of interest were drawn around the total liver and future remnant liver, and radioactivity was measured within these regions of interest (Fig. 1). For determination of uptake in the future remnant, the right border of the aorta (visible in the early phase of scintigraphy) plus 1 aortic diameter was taken as the border between segments 4 and $5 / 8$. The indentation formed by the round ligament was used as the border between segments 3 and 4 . For validation purposes, the regions of interest around the future remnant were drawn on the preoperative scan by 2 independent investigators with guidance from the surgical report. Interobserver variation was calculated. The total hepatic uptake rate was calculated as described by Ekman et al. (13). The rate of uptake in the liver was calculated using measured values obtained between 150 and $350 \mathrm{~s}$ after injection, to ensure that hepatic uptake calculations were performed during a phase of homogeneous distribution of the agent in the blood pool, before the rapid phase of hepatic excretion (13). To compensate for differences in liver functional requirements based on different patient weights and lengths, the total hepatic uptake rate $(\% / \mathrm{min})$ was divided by the body surface area (BSA) and was expressed as $\% / \mathrm{min} / \mathrm{BSA}$ (17). Uptake in the future remnant was calculated by dividing counts within the delineated future remnant by total hepatic counts within the same time frame and multiplying this factor by total hepatic uptake, corrected for BSA. Uptake in the future remnant was expressed as $\% / \mathrm{min} / \mathrm{BSA}$.

\section{CT Volumetry}

CT volumetry was performed as described by Shoup et al. (18). Patients underwent diagnostic, multiphase, contrast-enhanced abdominal CT before surgery. All CT was performed with a helical scanner (Philips). CT of the abdomen was performed to include the whole liver in 1 breath-hold, using a 5-mm collimation. The total liver, tumors, and future remnant were delineated on every image using portal and hepatic veins as landmarks for segmental division. Integrated software was used to calculate the volume of the total liver, total tumors, and future remnant after surgery. All delineations were made by an experienced radiologist. Tumor volume was subtracted from total hepatic volume. Total hepatic volume was corrected for weight and length differences and expressed as

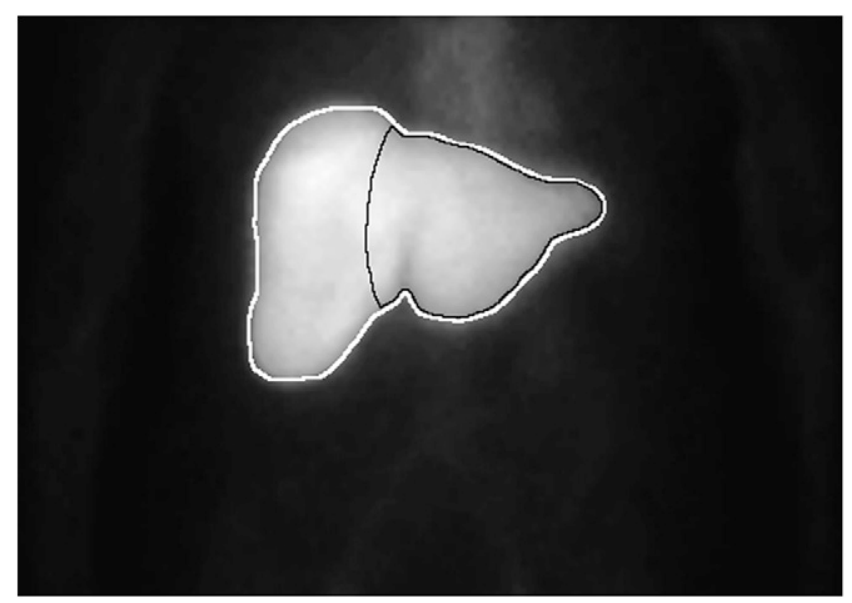

FIGURE 1. Example of summed hepatobiliary scintigraphy image of frames between 150 and $350 \mathrm{~s}$ after intravenous injection of $85 \mathrm{MBq}$ of ${ }^{99 \mathrm{~m} T c-m e b r o f e n i n . ~ R e g i o n s ~ o f ~ i n t e r e s t ~}$ are drawn around total liver (gray) and future remnant liver (black). Future-remnant-liver region of interest is drawn around left liver as if in the case of a right hemihepatectomy. 
$\mathrm{mL} / \mathrm{BSA}$. The volume of the future remnant was expressed as a percentage of total hepatic volume (18).

\section{Partial Liver Resection}

Three patients $(7 \%)$ underwent resection of 1 segment, 9 patients $(20 \%)$ underwent bisegmentectomy, 9 patients $(20 \%)$ underwent left hemihepatectomy, 18 patients (39\%) underwent right hemihepatectomy, and 7 patients $(15 \%)$ underwent extended right hemihepatectomy (including segment 4). Patients with hilar cholangiocarcinoma underwent (extended) hemihepatectomy combined with hilar resection and caudate lobe resection $(19,20)$. The median operation time was $349 \mathrm{~min}$ (range, 122-730 min), and median blood loss was 2,000 mL (range, 300-8,000 mL). Pringle's maneuver was applied 18 times (39\%), and total vascular exclusion was applied once (2\%) to control bleeding.

\section{Liver Failure}

Patients with liver failure were defined as having elevated bilirubin plasma levels $(>50 \mu \mathrm{mol} / \mathrm{L})$, prolonged prothrombin time ( $>15 \mathrm{~s}$ ), and increased plasma ammonia levels, combined with signs of hepatic encephalopathy or hepatorenal syndrome, requiring intensive care $(21,22)$.

\section{Statistical Analysis}

SPSS, version 11.5 for Windows (SPSS Inc.), was used for statistical analysis and for creating receiver-operating-characteristic (ROC) curves. Likelihood ratios (LRs) were used to assess posttest chances and cutoff values (23). The Student $t$ test or 1-way ANOVA, followed by Bonferroni posttest correction, was used to calculate differences in numeric data between 2 groups or more than 2 groups, respectively. The $\chi^{2}$ test was used for categoric data, and multivariate analysis was performed using logistic regression. Continuous data were expressed as mean $\pm \mathrm{SD}$ or median \pm range when nonparametric. All tests were considered at the $5 \%$ level of significance.

\section{RESULTS}

\section{Hepatobiliary Scintigraphy and CT Volumetry}

Total hepatic uptake was significantly lower in patients with parenchymal disease than in patients with healthy liver parenchyma, whereas total hepatic volume did not significantly differ between these groups (Table 1). No bias between the 2 observers was evident for delineation and calculation of uptake in the future remnant $(P=0.21)$, and $93 \%$ of values were within the limits of agreement (Fig. 2). Mean uptake and volume for the future remnant are shown

TABLE 1

99mTc-Mebrofenin Uptake and Volume for Total Liver According to Parenchymal Status

\begin{tabular}{|c|c|c|c|c|c|}
\hline Parameter & $n$ & $\begin{array}{c}\text { Uptake } \\
(\% / m i n / B S A)\end{array}$ & $P$ & $\begin{array}{c}\text { Volume } \\
\text { (mL/BSA) }\end{array}$ & $P$ \\
\hline Total & 46 & $7.5 \pm 1.8$ & & $958 \pm 218$ & \\
\hline $\begin{array}{l}\text { No parenchymal } \\
\text { disease }\end{array}$ & 29 & $8.1 \pm 1.6$ & 0.001 & $929 \pm 214$ & 0.25 \\
\hline $\begin{array}{l}\text { Parenchymal } \\
\text { disease }\end{array}$ & 17 & $6.3 \pm 1.7$ & & $1007 \pm 223$ & \\
\hline Data are mean \pm & SD. & & & & \\
\hline
\end{tabular}

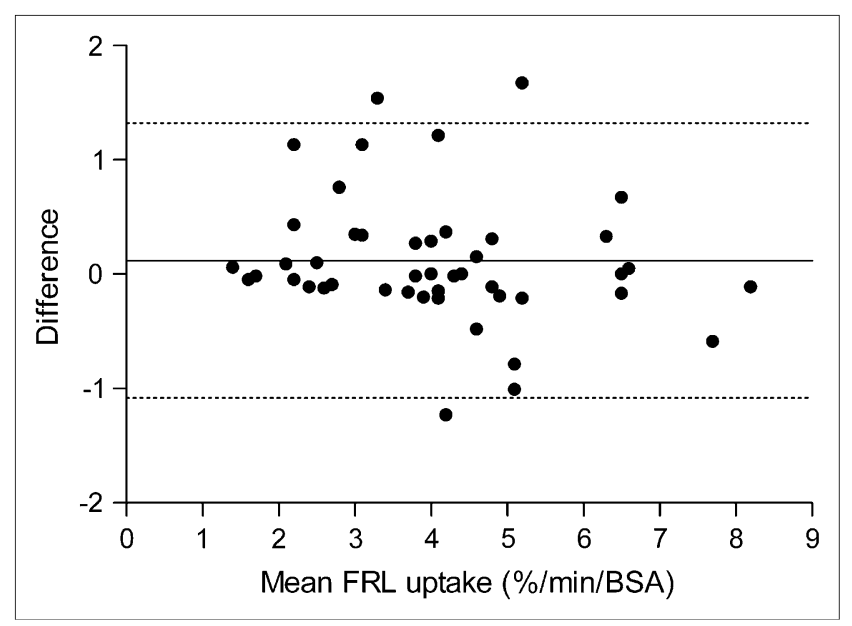

FIGURE 2. Bland-Altman plot showing mean uptake of $99 \mathrm{mTc}-$ mebrofenin in future remnant liver (FRL), as calculated in 46 hepatobiliary scintigraphy studies, versus differences in repeated calculations. Horizontal solid line indicates mean difference between the 2 calculations. Horizontal dashed lines indicate $95 \%$ limits of agreement (mean $\pm 1.96 \mathrm{SDs}$ ). No bias between the 2 observers was evident $(P=0.21$ with paired $t$ test). Ninety-three percent of values were within limits of agreement.

in Table 2 according to resection type and parenchymal status. Patients who underwent right or extended right hemihepatectomy had significantly lower uptake and volume for the future remnant than did all other patients. The mean size of the future remnant liver of extended right hemihepatectomy patients was also significantly less than that of right hemihepatectomy patients. Mean uptake in the future remnant was significantly lower in patients with parenchymal disease than in patients with healthy livers,

TABLE 2

99mTc-Mebrofenin Uptake and Volume for Future Remnant Liver According to Resection Type and Parenchymal Status

\begin{tabular}{|c|c|c|c|}
\hline Parameter & $n$ & $\begin{array}{c}\text { Uptake } \\
(\% / \mathrm{min} / \mathrm{BSA})\end{array}$ & Volume (\%) \\
\hline Total & 46 & $4.1 \pm 1.6$ & $51 \pm 20$ \\
\hline \multicolumn{4}{|l|}{ Resection type } \\
\hline $1+2$ segmentectomy & 12 & $5.3 \pm 1.7$ & $68 \pm 12$ \\
\hline Left hemihepatectomy & 9 & $4.8 \pm 1.6$ & $73 \pm 9$ \\
\hline Right hemihepatectomy & 18 & $3.4 \pm 0.9^{*}$ & $38 \pm 5^{\dagger}$ \\
\hline $\begin{array}{l}\text { Extended right } \\
\text { hemihepatectomy }\end{array}$ & 7 & $2.7 \pm 1.1^{\ddagger}$ & $27 \pm 10^{\dagger}$ \\
\hline No parenchymal disease & 29 & $4.5 \pm 1.6$ & $48 \pm 17$ \\
\hline Parenchymal disease & 17 & $3.4 \pm 1.5^{\S}$ & $57 \pm 24$ \\
\hline
\end{tabular}

*Significantly different from $1+2$ segmentectomy $(P=0.004)$.

${ }^{\dagger}$ Significantly different from all other groups $(P<0.05)$.

‡Significantly different from $1+2$ segmentectomy and left hemihepatectomy $(P<0.03)$.

${ }^{\S}$ Significantly different from no parenchymal disease $(P=0.02)$.

Data are mean \pm SD. Segmentectomies and bisegmentectomies were combined. 
whereas volume tended to be higher; this difference was not significant.

\section{Morbidity and Mortality}

The postoperative complications occurring most often were ascites, pulmonary failure, bleeding or repeated bleeding, and liver failure (Table 3). Liver failure occurred in 6 patients $(13 \%)$, of whom 4 had parenchymal disease $(67 \%)$ and 4 died in the hospital. Total morbidity and inhospital mortality rates were $61 \%(n=28)$ and $11 \%(n=$ 5 ), respectively (Table 4). In 2 of the 4 patients with liver failure who died, sepsis also had developed. In the fifth patient who died, sepsis associated with biliary anastomotic leakage had developed and eventually led to multiple organ failure. Besides the 5 patients who died, 4 other patients also needed treatment in the intensive care unit because of sepsis $(n=2)$, cardiac failure $(n=1)$, or pulmonary failure $(n=1)$. Laparotomies were repeated to control bleeding $(n=4)$ or to drain infected ascites and wash the abdominal cavity $(n=3)$. Mean uptake and volume for the future remnant were calculated according to the different outcome parameters (Table 5). Uptake was significantly lower in patients in whom liver failure developed and in patients who experienced liver failure-related mortality. Volume tended to be lower in patients with morbidity, liver failure, and mortality, but this difference was not significant.

\section{ROC Analysis}

To further examine the significant relationship between uptake in the future remnant and the outcome parameters of liver failure and liver failure-related mortality, we performed ROC analysis and determined cutoff values (Fig. 3). ROC analysis of the volume of the future remnant was also performed; areas under the curve for which the $95 \%$ confidence interval crossed $50 \%$ indicated no significant accuracy. Cutoff values were assessed on the following bases: First, the chance that a negative outcome would develop while the test result was above the cutoff value (a "negative test") needed to be as low as possible. Second, when the

\section{TABLE 3}

Postoperative Complications

\begin{tabular}{lr}
\hline \multicolumn{1}{c}{ Complication } & $n$ \\
\hline Ascites & $10(22 \%)$ \\
Pulmonary failure & $7(15 \%)$ \\
Bleeding or repeated bleeding & $7(15 \%)$ \\
Liver failure & $6(13 \%)$ \\
Infected ascites/peritonitis & $6(13 \%)$ \\
Abdominal fluid collection & $5(11 \%)$ \\
Sepsis & $4(9 \%)$ \\
Bile leakage & $4(9 \%)$ \\
Miscellaneous & $24(52 \%)$
\end{tabular}

*Intraabdominal abscess, wound infection/rupture, pneumonia, renal failure, urinary tract infection, delirium, liver abscess/necrosis, pleural fluid, cholangitis, and cardiac failure.
TABLE 4

Outcome Characteristics

\begin{tabular}{lc}
\hline \multicolumn{1}{c}{ Characteristic } & $n$ \\
\hline Morbidity $(n)$ & $28(61 \%)$ \\
Median hospital stay $(d)$ & 15 \\
Range of hospital stay $(d)$ & $7-79$ \\
Repeated laparotomy $(n)$ & $7(15 \%)$ \\
Treatment in intensive care unit $(n)$ & $9(20 \%)$ \\
In-hospital mortality $(n)$ & $5(11 \%)$ \\
\hline
\end{tabular}

test result was below the cutoff value (a "positive test"), the decision not to perform the operation needed to be well supported. In other words, the LR of a negative test (LR-) needed to be as low as possible while the LR of a positive test $(\mathrm{LR}+)$ was kept well above 5. These cutoff values are encircled in Figure 3 and were found to be $2.5 \% / \mathrm{min} / \mathrm{BSA}$ for liver failure $(\mathrm{LR}-=0.2 ; \mathrm{LR}+=8.3)$ and $2.2 \% / \mathrm{min} /$ BSA for liver failure-related mortality $(\mathrm{LR}-=0.3 ; \mathrm{LR}+=$ 11). Patients with uptakes above $2.5 \% / \mathrm{min} / \mathrm{BSA}$ and $2.2 \% /$ $\mathrm{min} / \mathrm{BSA}$ in the future remnant had a $3 \%$ chance that liver failure and liver failure-related mortality, respectively, would develop (Table 6). Patients with uptake below $2.5 \% / \mathrm{min} / \mathrm{BSA}$ in the future remnant had a $56 \%$ chance that liver failure would develop, whereas patients with uptake below $2.2 \% / \mathrm{min} / \mathrm{BSA}$ had a $50 \%$ chance that liver failure-related mortality would occur. Beneath Table 6, 2 by 2 tables are shown for each cutoff value.

\section{Univariate and Multivariate Analyses}

To examine whether other preoperative and intraoperative factors were also associated with the outcome parameters and to identify potential confounders, we performed univariate and multivariate analyses (Table 7). Factors besides uptake that were evaluated in univariate analysis were age, sex, diagnosis, chronic liver disease, resection type, operation time, blood loss, and the following preoperative laboratory assessments: aspartate aminotransferase, alanine aminotransferase, lactate dehydrogenase, albumin, bilirubin, alkaline phosphatase, $\gamma$-glutamyl transferase, prothrombin time, activated partial thromboplastin time, antithrombin III, and ICG clearance rate. Only factors found to be significant on univariate analysis are shown in Table 7.

In multivariate analysis, no factors were associated with morbidity. Operation time was the only factor that was significantly associated with mortality. Uptake in the future remnant was the only factor that was significantly associated with liver failure. No factor was significantly associated with liver failure-related mortality.

\section{DISCUSSION}

Accurate measurement of liver function before liver resection is crucial in the assessment of resectability and hepatic functional reserve, especially in patients who require a large resection and patients with underlying parenchymal liver 
TABLE 5

99mTc-Mebrofenin Uptake and Volume for Future Remnant Liver According to Outcome Parameters

\begin{tabular}{|c|c|c|c|c|c|c|}
\hline Parameter & Outcome & $n$ & Uptake (\%/min/BSA) & $P$ & Volume (\%) & $P$ \\
\hline \multirow[t]{2}{*}{ Morbidity } & Yes & 28 & $3.9 \pm 1.4$ & 0.36 & $48 \pm 20$ & 0.14 \\
\hline & No & 18 & $4.3 \pm 1.9$ & & $57 \pm 20$ & \\
\hline \multirow[t]{2}{*}{ Mortality } & Yes & 5 & $2.9 \pm 1.4$ & 0.09 & $45 \pm 27$ & 0.50 \\
\hline & No & 41 & $4.2 \pm 1.5$ & & $52 \pm 20$ & \\
\hline \multirow[t]{2}{*}{ Liver failure } & Yes & 6 & $2.3 \pm 0.6$ & 0.003 & $42 \pm 25$ & 0.26 \\
\hline & No & 40 & $4.3 \pm 1.5$ & & $52 \pm 20$ & \\
\hline \multirow[t]{2}{*}{ Liver failure-related mortality } & Yes & 4 & $2.3 \pm 0.7$ & 0.02 & $45 \pm 31$ & 0.51 \\
\hline & No & 42 & $4.2 \pm 1.6$ & & $52 \pm 19$ & \\
\hline
\end{tabular}

Data are mean $\pm \mathrm{SD}$

disease (24). In this study, the accuracy for assessing the risk of morbidity, liver failure, and mortality was measured before partial liver resection, comparing uptake in the future remnant liver on hepatobiliary scintigraphy with volume of the future remnant on CT volumetry. Uptake was accurate for determining an increased or decreased risk that liver failure and liver failure-related mortality would develop, whereas volume was not. To our knowledge, this was the first study that compared uptake and volume for risk assessment after partial liver resection.

Apart from hepatobiliary scintigraphy, several other liver function tests have been described in the literature. However, the predictive value of these tests has usually not been described. The Child-Pugh classification, based on clinical symptoms of liver failure (ascites/encephalopathy) and laboratory analysis of liver function (albumin, bilirubin, and prothrombin time), can be used to identify high-risk patients (25). Other, more specific, liver function tests such as the galactose elimination test and ICG clearance rate can complement the clinical Child-Pugh classification $(26,27)$. One study, by Nonami et al. (27), found that ICG clearance rate correlated with morbidity and mortality after partial liver resection, but this finding was not supported in the present study. One explanation is that in the present study, as opposed to the study by Nonami et al., both small and large resections were performed, and not only hepatocellular carcinoma patients were evaluated. Furthermore, when only total hepatic function is measured, as in the ICG test, and not specifically the function of the future remnant, no conclusion can be made about the volume of liver parenchyma that can safely be resected in any individual patient.

Another method to measure functional distribution in the liver is scintigraphy with ${ }^{99 m}$ Tc-labeled galactosyl human serum albumin (GSA). This receptor-targeted liver function test (28-31) correlated well with ICG clearance and conventional liver function parameters $(28,30)$. In several studies, the test also seems to have predictive value for short-term outcome (29,31). However, ROC analysis was never performed in these studies. Furthermore, ${ }^{99 \mathrm{~m} T c-G S A}$ for clinical use is available only in Japan, not in Europe or the United States.
CT volumetric analysis of the future remnant correlated with outcome after resection in 3 studies $(7,18,32)$. These and other studies have determined that a minimal remnant volume of $25 \%-30 \%$ is necessary to maintain adequate function after partial resection of healthy livers $(9,33)$. In the present study, however, no significant correlation between volume measured with CT volumetry and postoperative morbidity, liver failure, or mortality was found, although trends were present. This discrepancy may be explained as follows. The 3 mentioned studies dealt with homogeneous groups of patients. In 2 of the studies $(7,18)$, only patients without parenchymal disease were evaluated. In the third study (32), only patients with hepatocellular carcinoma who underwent large (no less than bisegmentectomy) resections were evaluated. Therefore, in these studies, patients with approximately the same level of liver function were selected. In the present study, patients with and without parenchymal disease were evaluated. The volume of the future remnant liver was about the same between patients with and without parenchymal disease, whereas uptake ratios were significantly lower in patients with parenchymal disease. When only patients without parenchymal disease are evaluated in the present study, volume is significantly lower in patients in whom posthepatectomy liver failure developed than in other patients $(28 \% \pm 5 \%$ vs. $49 \% \pm 17 \%, P=0.02)$. Therefore, although measurement of volume may be valuable in patient populations without much variation in liver function, the advantage of measuring uptake is that it can be used for risk assessment in patient populations with or without parenchymal disease.

An earlier study found a good correlation between the rates of total hepatic uptake and ICG clearance (15). Furthermore, another study found a strong correlation between the preoperative rate of uptake in the future remnant and the actually measured uptake in the remnant $1 \mathrm{~d}$ after surgery ( $r=0.95 ; P<0.001$ ) (16). Consistent delineation of the future remnant can readily be learned, especially when performed on hepatobiliary scintigraphy after being performed on CT. The 2 experienced observers in this study reached acceptable agreement for measurement of uptake in the future remnant. Visualization of functional distribution on 

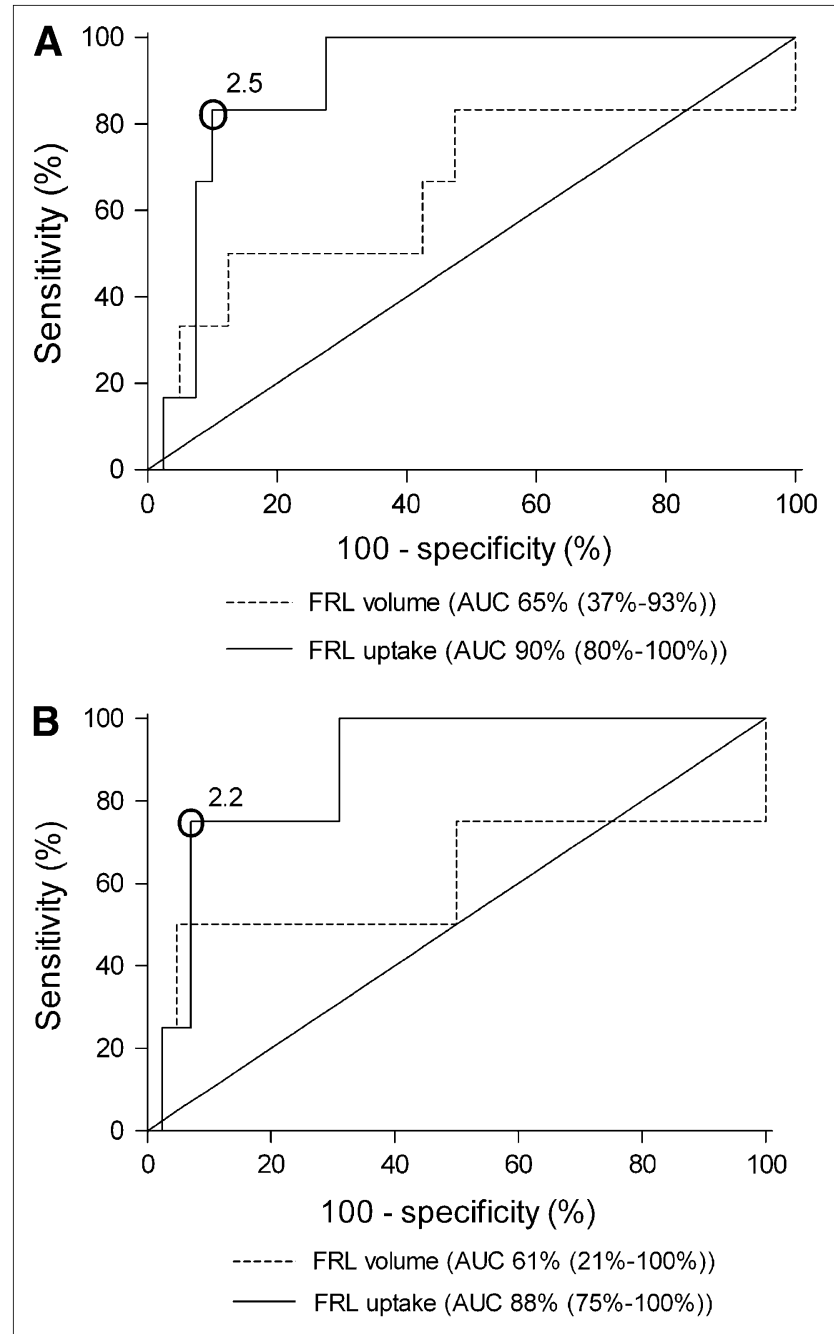

FIGURE 3. ROC curves for liver failure (A) and liver failurerelated mortality $(B)$. Test results were considered positive when below cutoff value. Continuous staircase line represents uptake in future remnant liver (FRL), dotted staircase line represents $\mathrm{FRL}$ volume, and straight diagonal line is reference line $(y=x)$. Area-under-curve (AUC) percentages are given beneath each graph, with $95 \%$ confidence interval between brackets. Cutoff values are encircled and noted.

other techniques such as SPECT might further increase interobserver and intraobserver consistency. The latter technique produces more realistic, 3-dimensional images of the liver, enabling more accurate segmental delineation.

In this study, in-hospital morbidity and mortality were high, compared with data reported in the literature (1-4). There are 2 explanations for the relatively high mortality rate. First, $37 \%$ of patients had parenchymal disease resulting in compromised liver function. Four of 6 patients with liver failure had parenchymal disease. Second, a relatively large proportion of patients had hilar cholangiocarcinoma (28\%). These patients require large resections in combination with biliary anastomoses, with a notoriously increased risk of postoperative morbidity and mortality (19). Reported in-hospital mortality rates after resection for
TABLE 6

Diagnostic Characteristics of Cutoff Values

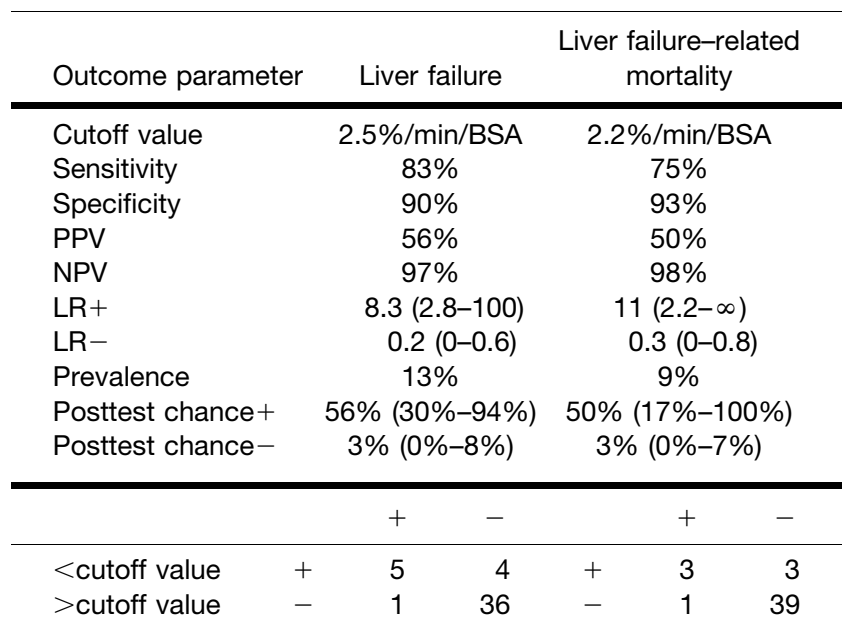

$\mathrm{PPV}=$ positive predictive value; NPV $=$ negative predictive value; posttest chance $+=$ posttest chance when test is positive; posttest chance $-=$ posttest chance when test is negative.

Data in parentheses are $95 \%$ confidence intervals. Tests were considered positive when below cutoff value. $2 \times 2$ tables are shown beneath the table.

hilar cholangiocarcinoma vary from $0 \%$ to $15 \%(19,34-36)$. Overall, 25 (54\%) of 46 patients required (extended) right hemihepatectomy, which resulted in an average volume of $35 \% \pm 8 \%$ and an average uptake of $3.2 \% \pm 0.9 \% / \mathrm{min} /$ BSA for the future remnant in these patients. Notwithstanding the fact that postoperative morbidity and mortality were considerable, it did furnish necessary power to this study, in which risk assessment was the primary goal.

Uptake in the future remnant and operation time were the only independent factors associated with outcome in multivariate analysis. A correlation between operation time and short-term outcome after partial liver resection has also been reported by others ( $I$ ). Because liver resections can prove more demanding than anticipated on preoperative imaging, there will always be an unpredictable element in the risk assessment of patients requiring an extensive liver resection. This factor will translate into a prolonged surgical procedure. Although no factors were significantly associated with liver failure-related mortality, uptake in the future remnant had the lowest $P$ value (0.19). Diagnosis was a confounding factor, because three fourths of the patients who died with liver failure had hilar cholangiocarcinoma.

\section{CONCLUSION}

In a population including patients with parenchymal disease and hilar cholangiocarcinoma, preoperative measurement of ${ }^{99 \mathrm{~m}} \mathrm{Tc}$-mebrofenin uptake in the future remnant liver on functional hepatobiliary scintigraphy proved more valuable than measurement of the volume of the future remnant in the assessment of the posthepatectomy risk of liver failure and liver failure-related mortality. 
TABLE 7

Univariate and Multivariate Analyses of Pre- and Perioperative Factors Associated with Outcome Parameters

\begin{tabular}{|c|c|c|c|c|}
\hline \multirow[b]{2}{*}{ Outcome parameter } & \multirow[b]{2}{*}{ Univariate analysis $(P)$} & \multicolumn{3}{|c|}{ Multivariate analysis } \\
\hline & & $P$ & Odds ratio & 95\% confidence interval \\
\hline \multicolumn{5}{|l|}{ Morbidity } \\
\hline Diagnosis & $0.02^{*}$ & 0.27 & 0.38 & $0.05-2.34$ \\
\hline Resection type & $0.02^{\dagger}$ & 0.08 & 0.30 & $0.07-1.28$ \\
\hline Operation time & 0.01 & 0.23 & 1.00 & $0.99-1.00$ \\
\hline Mortality (operation time) & 0.02 & 0.04 & 0.99 & $0.99-1.00$ \\
\hline \multicolumn{5}{|l|}{ Liver failure } \\
\hline Uptake & 0.01 & 0.03 & 3.96 & $1.17-13.4$ \\
\hline Diagnosis & $0.04^{*}$ & 0.36 & 0.37 & $0.04-3.12$ \\
\hline \multicolumn{5}{|c|}{ Liver failure-related mortality } \\
\hline Uptake & 0.02 & 0.19 & 80.8 & $0.12-53,700$ \\
\hline Diagnosis & $0.04^{*}$ & 0.40 & 102 & $0.00-44,200$ \\
\hline Operation time & 0.01 & 0.27 & 0.96 & $0.89-1.04$ \\
\hline
\end{tabular}

*Hilar cholangiocarcinoma patients were compared with other patients combined.

${ }^{\dagger}$ (Extended) hemihepatectomy patients were compared with other patients combined.

Factors that were evaluated in univariate analysis were $99 \mathrm{mTc}$-mebrofenin uptake, age, sex, diagnosis, chronic liver disease, resection type, operation time, blood loss, preoperative aspartate aminotransferase, alanine aminotransferase, lactate dehydrogenase, albumin, bilirubin, alkaline phosphatase, $\gamma$-glutamyl transferase, prothrombin time, activated partial thromboplastin time, antithrombin III, and ICG clearance rate. Only significant factors in univariate analysis are shown.

\section{ACKNOWLEDGMENT}

We thank Dr. Dirk Ubbink, Department of Surgery/ Clinical Epidemiology and Biostatistics, Academic Medical Center, for his statistical advice.

\section{REFERENCES}

1. Jarnagin WR, Gonen M, Fong Y, et al. Improvement in perioperative outcome after hepatic resection: analysis of 1,803 consecutive cases over the past decade. Ann Surg. 2002;236:397-406.

2. Poon RT, Fan ST, Lo CM, et al. Extended hepatic resection for hepatocellular carcinoma in patients with cirrhosis: is it justified? Ann Surg. 2002;236:602-611.

3. Belghiti J, Hiramatsu K, Benoist S, Massault P, Sauvanet A, Farges O. Seven hundred forty-seven hepatectomies in the 1990s: an update to evaluate the actual risk of liver resection. J Am Coll Surg. 2000;191:38-46.

4. Nagino M, Kamiya J, Uesaka K, et al. Complications of hepatectomy for hilar cholangiocarcinoma. World J Surg. 2001;25:1277-1283.

5. Das BC, Isaji S, Kawarada Y. Analysis of 100 consecutive hepatectomies: risk factors in patients with liver cirrhosis or obstructive jaundice. World J Surg. 2001;25:266-272.

6. Farges O, Malassagne B, Flejou JF, Balzan S, Sauvanet A, Belghiti J. Risk of major liver resection in patients with underlying chronic liver disease: a reappraisal. Ann Surg. 1999;229:210-215.

7. Yigitler C, Farges O, Kianmanesh R, Regimbeau JM, Abdalla EK, Belghiti J. The small remnant liver after major liver resection: how common and how relevant? Liver Transpl. 2003;9(suppl):S18-S25.

8. Vauthey JN, Chaoui A, Do KA, et al. Standardized measurement of the future liver remnant prior to extended liver resection: methodology and clinical associations. Surgery. 2000;127:512-519.

9. Makuuchi M, Thai BL, Takayasu K, et al. Preoperative portal embolization to increase safety of major hepatectomy for hilar bile duct carcinoma: a preliminary report. Surgery. 1990;107:521-527.

10. Farges O, Belghiti J, Kianmanesh R, et al. Portal vein embolization before right hepatectomy: prospective clinical trial. Ann Surg. 2003;237:208-217.

11. Wakabayashi H, Ishimura K, Okano K, et al. Application of preoperative portal vein embolization before major hepatic resection in patients with normal or abnormal liver parenchyma. Surgery. 2002;131:26-33.

12. Heyman S. Hepatobiliary scintigraphy as a liver function test. J Nucl Med. 1994; $35: 436-437$
13. Ekman M, Fjalling M, Friman S, Carlson S, Volkmann R. Liver uptake function measured by IODIDA clearance rate in liver transplant patients and healthy volunteers. Nucl Med Commun. 1996;17:235-242.

14. Hendrikse $\mathrm{NH}$, Kuipers F, Meijer C, et al. In vivo imaging of hepatobiliary transport function mediated by multidrug resistance associated protein and P-glycoprotein. Cancer Chemother Pharmacol. 2004;54:131-138.

15. Erdogan D, Heijnen BH, Bennink RJ, et al. Preoperative assessment of liver function: a comparison of ${ }^{99 \mathrm{~m}} \mathrm{Tc}-\mathrm{mebrofenin}$ scintigraphy with indocyanine green clearance test. Liver Int. 2004;24:117-123.

16. Bennink RJ, Dinant S, Erdogan D, et al. Preoperative assessment of postoperative remnant liver function using hepatobiliary scintigraphy. $\mathrm{J} \mathrm{Nucl} \mathrm{Med}$. 2004;45:965-971.

17. Du Bois D, Du Bois EF. A formula to estimate the approximate surface area if height and weight be known. Arch Intern Med. 1916;1:863-871.

18. Shoup M, Gonen M, D'Angelica M, et al. Volumetric analysis predicts hepatic dysfunction in patients undergoing major liver resection. J Gastrointest Surg. 2003;7:325-330.

19. Dinant S, Gerhards MF, Rauws EAJ, Busch ORC, Gouma DJ, van Gulik TM. Improved outcome of resection of hilar cholangiocarcinoma (Klatskin tumour). Ann Surg Oncol. 2006;13:872-880.

20. Dinant S, Gerhards MF, Busch ORC, Obertop H, Gouma DJ, van Gulik TM. The importance of complete excision of the caudate lobe in resection of hilar cholangiocarcinoma. HPB. 2005;7:263-267.

21. Lee WM. Acute liver failure. Am J Med. 1994;96(suppl):2S-9S.

22. Fingerote RJ, Bain VG. Fulminant hepatic failure. Am J Gastroenterol. 1993; $88: 1000-1010$

23. Archibald S, Bhandari M, Thoma A. Users' guides to the surgical literature: how to use an article about a diagnostic test. Can J Surg. 2001;44:17-23.

24. Schneider PD. Preoperative assessment of liver function. Surg Clin North Am. 2004;84:355-373.

25. Reisman Y, van Dam GM, Gips CH, Lavelle SM, Euricterus PM. Survival probabilities of Pugh-Child-PBC classified patients in the euricterus primary biliary cirrhosis population, based on the Mayo clinic prognostic model. Euricterus Project Management Group. Hepatogastroenterology. 1997;44:982-989.

26. Redaelli CA, Dufour JF, Wagner M, et al. Preoperative galactose elimination capacity predicts complications and survival after hepatic resection. Ann Surg. 2002;235:77-85.

27. Nonami T, Nakao A, Kurokawa T, et al. Blood loss and ICG clearance as best prognostic markers of post-hepatectomy liver failure. Hepatogastroenterology. 1999;46:1669-1672.

28. Onodera Y, Takahashi K, Togashi T, Sugai Y, Tamaki N, Miyasaka K. Clinical assessment of hepatic functional reserve using ${ }^{99 m}$ Tc DTPA galactosyl 
human serum albumin SPECT to prognosticate chronic hepatic diseases: validation of the use of SPECT and a new indicator. Ann Nucl Med. 2003;17: 181-188.

29. Kokudo N, Vera DR, Tada K, et al. Predictors of successful hepatic resection: prognostic usefulness of hepatic asialoglycoprotein receptor analysis. World $\mathrm{J}$ Surg. 2002;26:1342-1347.

30. Mitsumori A, Nagaya I, Kimoto S, et al. Preoperative evaluation of hepatic functional reserve following hepatectomy by technetium-99m galactosyl human serum albumin liver scintigraphy and computed tomography. Eur J Nucl Med. 1998;25:1377-1382.

31. Hwang EH, Taki J, Shuke N, et al. Preoperative assessment of residual hepatic functional reserve using ${ }^{99 \mathrm{~m}}$ Tc-DTPA-galactosyl-human serum albumin dynamic SPECT. J Nucl Med. 1999;40:1644-1651.
32. Shirabe K, Shimada M, Gion T, et al. Postoperative liver failure after major hepatic resection for hepatocellular carcinoma in the modern era with special reference to remnant liver volume. J Am Coll Surg. 1999;188:304-309.

33. Abdalla EK, Hicks ME, Vauthey JN. Portal vein embolization: rationale, technique and future prospects. Br J Surg. 2001;88:165-175.

34. Jarnagin WR, Fong Y, DeMatteo RP, et al. Staging, resectability, and outcome in 225 patients with hilar cholangiocarcinoma. Ann Surg. 2001;234:507-517.

35. Nagino M, Kamiya J, Arai T, Nishio H, Ebata T, Nimura $Y$. One hundred consecutive hepatobiliary resections for biliary hilar malignancy: preoperative blood donation, blood loss, transfusion, and outcome. Surgery. 2005;137:148-155.

36. Kondo S, Hirano S, Ambo Y, et al. Forty consecutive resections of hilar cholangiocarcinoma with no postoperative mortality and no positive ductal margins: results of a prospective study. Ann Surg. 2004;240:95-101. 